\title{
COLLISION AVOIDANCE TECHNOLOGY OF INTELLIGENT VEHICLE SYSTEM BASED ON MULTI-SENSOR FUSION
}

\author{
Yunqiang $Y u$ \\ Institute of Control Technology, Wuxi Institute of Technology, Wuxi 214125, China \\ E-mail: jiangsuwxyyq@163.com
}

\begin{abstract}
In order to reduce the occurrence of traffic accidents, aiming at the shortcomings of using single sensor for target recognition in smart vehicle collision avoidance in the past, such as low perception range and high recognition error, the collision avoidance technology of smart vehicle system based on multi-sensor fusion is deeply studied. Arduino is the main control unit of smart vehicle collision avoidance system, Linux is the development platform, and HC-SR04 ultrasonic ranging module is used. $2 \mathrm{~cm}$ to $400 \mathrm{~cm}$ non-contact distance sensing is realized, and the detection distance is adjusted by the potentiometer knob in the infrared sensor module; after building the intelligent vehicle motion model, collision avoidance is realized by using the combination mode of ultrasonic single-point obstacle avoidance and infrared double-way crossobstacle avoidance. In the process of collision avoidance, Mahalanobis distance is introduced to match the observed value of vehicle target sequence, and then the joint probability data association algorithm is applied to fuse. The data of ultrasonic sensor and infrared sensor are used to realize automatic collision avoidance of intelligent vehicle based on multi-sensor fusion. The results show that the collision avoidance technology is not affected by the shape of obstacles and has high collision avoidance rate. It can respond to braking instructions with high efficiency and precision, enhance the collision avoidance performance of intelligent vehicles, and has high accuracy of data fusion. It can effectively realize multi-aspect detection and omni-directional automatic collision avoidance of intelligent vehicles.
\end{abstract}

Keywords: Multi-Sensor; Fusion; Intelligent Vehicle; Collision Avoidance; Ultrasonic Ranging; Infrared Sensing.

\section{Introduction}

In recent years, the rapid development of the automobile industry and the improvement of national purchasing power have led to the increase of automobile sales, but also inevitably increased the possibility of traffic accidents (Lu et al. 2017). From the point of view of protecting personal safety and reducing the possibility of traffic accidents, it is very important to develop a vehicle active collision avoidance system which can remind drivers to take corresponding measures in time when two vehicles are in danger of collision, and even actively intervene intelligent vehicles and automatically take emergency braking measures to effectively avoid collision (Rong et al. 2018). As an advanced active safety technology, collision warning/avoidance (CW/CA) is of great significance to improve traffic safety (Li 2017). When the collision avoidance system detects potential collision hazards, an appropriate alarm mode will be triggered; if the driver does not make timely and effective operation of the alarm signal, the collision avoidance system will automatically take over vehicle control and avoid or mitigate collision accidents by active braking (Li \& Xing 2017). As early as the 1990s, driven by the American AHS (Automated Highway System) project, active collision avoidance system began to be widely studied in China and abroad, focusing on the design of safe distance model (Fei \& Li 2016). Representative models such as MAZDA model, HONDA model, Berkeley model, Jaguar model and NHSTA model have been proposed (Zheng et al. 2017). The University of Michigan evaluated the missing and false alarm rates of the five models based on ICC FOT database. Among them, the NHSTA model of National Highway Transportation Safety Administration performs best, but the alarm accuracy is only $23 \%$. Therefore, there is still much room for improvement of the existing model algorithm (Jia \& Cebon 2016).

Robots have made great progress in technology from the initial demonstration of imitating robots to the present intelligent robots with perceptual functions (Husler et al. 2016). As a four-wheel drive perceptual robot, intelligent vehicle is flexible and easy to operate. It can integrate various precise sensor data processing modules on the body. 
Its obstacle avoidance function ensures the automatic adjustment of the direction of intelligent vehicle in the course of moving and avoids collision. It is an important part of intelligent vehicle (Jie et al. 2017). Scholars in China and abroad have proposed various vehicle collision warning methods and systems from different angles. At present, intelligent vehicle collision avoidance methods and systems which have been applied in engineering field generally identify obstacles in front of them through machine vision, radar, infrared or ultrasound, and then make collision avoidance decisions. Finally, the decision results are output to the actuator or early warning human machine interface (human machine intel). Rface, HMI). However, single sensor will be affected by various adverse environmental factors, resulting in a significant reduction in the ability of target recognition in specific scenarios (Johansen et al. 2016). At present, most intelligent vehicles adopt single sensor obstacle avoidance method, but single sensor obstacle avoidance has some shortcomings such as difficulty in obstacle detection and low success rate of obstacle avoidance (Lin \& Saripalli 2017). Therefore, this paper studies the collision avoidance technology of intelligent vehicle system based on multi-sensor fusion, using the mode of combination of single-point ultrasonic obstacle avoidance and infrared double-way cross obstacle avoidance, to achieve multi-dimensional detection and omni-directional automatic collision avoidance.

\section{Materials and Methods}

\subsection{Hardware design of collision avoidance system}

Intelligent vehicle collision avoidance system based on multi-sensor fusion uses multi-module coordination to make it have high adaptive ability.

The block diagram of the whole system module is shown in Figure 1. Its overall module includes: power supply module, ultrasonic sensor module, infrared sensor module, motor drive module, core control module and so on. In the process of intelligent vehicle moving forward, the sensor module continuously detects the distance of surrounding obstacles. When obstacles affect the movement of intelligent vehicles, the sensor returns the measured data to the core control module, which steers through built-in program settings to avoid obstacles, thus realizing the obstacle avoidance of intelligent vehicles (Bila et al. 2017).

The block diagram of the whole system module is shown in Figure 1.

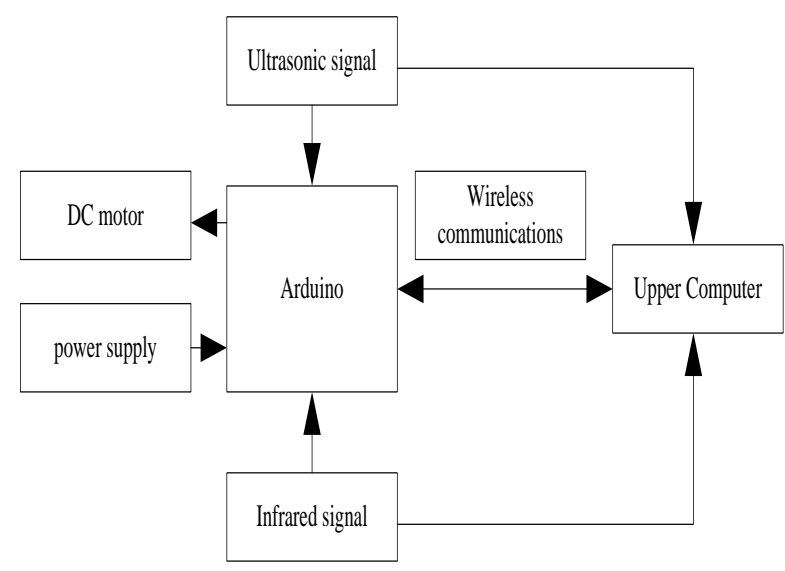

Figure 1: System block diagram

\subsubsection{Core control module}

The core control module adopts Arduino Yun control chip. The core of the chip is ATmega32u4 MCU, and it has embedded Linux machine. In addition, it includes the following parts: a 9VDC input, a USB interface, 14 digital I/O ports, 6 analog I/O ports, a 5VDC output and a 3.3VDC output. Arduino Yun is used as the microcontroller of the intelligent vehicle. The Linux system in Arduino Yun saves the design of peripheral devices and realizes WiFi communication with the host computer. Under special circumstances, the host computer can directly control the action of the intelligent vehicle.

\subsubsection{Motor module}

Intelligent vehicles are driven by dual DC motors, which control the forward, backward or left-right steering of the vehicle by controlling the left and right DC motors. The motor module is shown in Figure 4. The DC motor is driven by the DC motor chip L298N. The maximum output current of the two-way full-bridge motor driver chip L298 is 4A, which has over-temperature protection function and high noise rejection ratio. The two outputs can meet the driving requirements of the left and right DC motors (Sabattini et al. 2017).

The operation state of the motor can be controlled by the level input of L298N3 input ports. The working state of the motor is shown in Table 1.

Table 1. DC motor operating status

\begin{tabular}{|c|c|c|c|}
\hline ENA & IN1 & IN2 & Operating status \\
\hline 0 & $\mathrm{X}$ & $\mathrm{X}$ & stop \\
\hline 1 & 1 & 0 & Positive turn \\
\hline 1 & 0 & 1 & reverse \\
\hline 1 & 1 & 1 & Brake \\
\hline 1 & 0 & 0 & stop \\
\hline
\end{tabular}


Because chip L298N does not provide the control mode of motor speed, the PWM signal of driving motor is adjusted by Arduino control board to change the output power of the motor, so as to control the speed of left and right motor.

When the motor runs, when it suddenly changes from running state to stopping state, or from clockwise state to counter-clockwise state, a large reverse current will be formed. For this reason, a diode protection circuit is added to the circuit. When the reverse current is generated, the current will be drained through the diode to protect the safety of the chip.

\subsubsection{Ultrasound sensor module}

The collision avoidance system adopts HC-SR04 ultrasonic ranging module, which can provide noncontact distance sensing function of $2 \mathrm{~cm}$ to $400 \mathrm{~cm}$ with ranging accuracy up to $3 \mathrm{~mm}$. The module includes ultrasonic transmitter, receiver and control circuit. During detection, the transmitter converts the electric vibration energy of the ceramic oscillator into ultrasonic energy and radiates it into the air (Funke et al. 2017). The receiver receives the ultrasonic wave to produce mechanical vibration, and converts it into electrical energy, which serves as the output of the receiver of the sensor to detect the transmitted ultrasonic wave. The receiving circuit consists of TL074 operational amplifier and R40 ultrasonic receiver.

In ultrasonic testing, the module automatically sends eight $40 \mathrm{kHz}$ square wave levels, and automatically detects whether there is a return signal (Mylvaganam et al. 2017). Once the return of the signal is detected, the ultrasonic signal is output again, and a high level is output through the I/O port.

The duration of the high level is the time $t$ of the second ultrasonic wave from transmission to return.

Thus, the distance $L$ between the acoustic transmitter and the obstacle can be obtained. The relationship between the distance and the obstacle is as follows:

$$
L=C \mid t / 2
$$

In the formula, $C$ is the propagation speed of ultrasonic wave in air.

\subsubsection{Infrared sensor module}

Infrared sensor module has strong adaptability to infrared radiation in different environmental light. It emits a certain frequency of infrared radiation with the receiving tube. When the detection direction encounters obstacles (reflecting surface), the infrared reflects back and is received by the receiving tube. After processing by the comparator circuit, the green indicator lights up, and the signal output interface outputs digital signal (a low-power).
The detection distance can be adjusted by potentiometer knob. The effective distance is 2-30 $\mathrm{cm}$ and the working voltage is $3.3 \mathrm{~V}-5 \mathrm{~V}$. The detection distance of the sensor can be adjusted by potentiometer, which has the characteristics of small interference, easy assembly and easy use.

\subsection{Motion model of intelligent vehicle}

The intelligent vehicle adopts four-wheel drive mode and is controlled by two left and right motors.

The left motor controls the left two wheels and the right motor controls the right two wheels. Each motor is connected with the shaft through variable speed gears to control the rotation of the front and rear wheels on the corresponding side.

The kinematics model of the intelligent vehicle is shown in Figure 2.

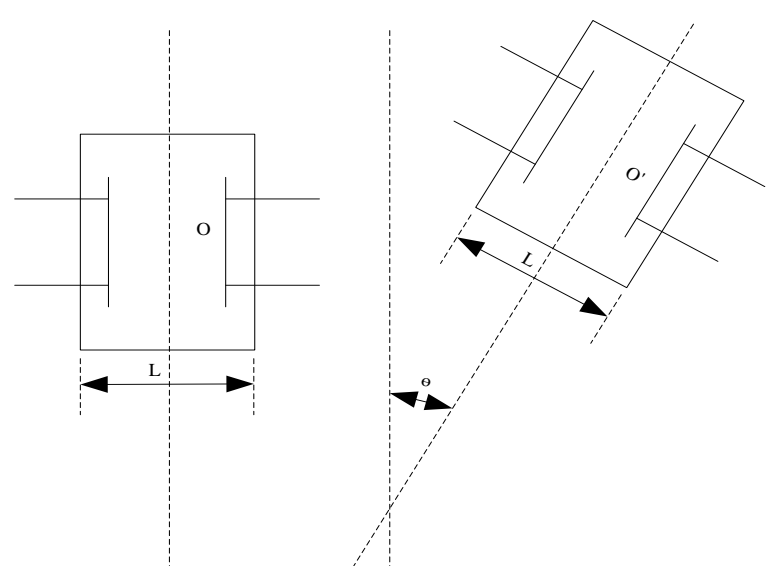

Figure 2: Motor model of smart vehicle

Assuming that the wheel is in an ideal rolling state, at a certain moment, the central coordinate point of the intelligent vehicle is point 0 . After $\Delta t$ time, the central point of the vehicle moves to point $0^{\prime}$, the deflection angle is $\theta$, the linear speed of the left wheel is $V_{1}$, the linear speed of the right wheel is $V_{2}$, the instantaneous curvature radius of $R$ is the center, and the distance between the left and right wheels is $L$, then the instantaneous curvature radius of the left wheel is $R+L / 2$, and the instantaneous curvature radius of the right wheel is $R-L / 2$. The angular speed of wheel rotation is $\omega$, so the speed of both left and right wheels of a vehicle can be expressed as follows:

$$
\begin{aligned}
& V_{1}=\omega(R+L / 2) \\
& V_{2}=\omega(R-L / 2)
\end{aligned}
$$

In the model, $\omega$ is the angular speed of wheel rotation.

From formulas (2) and (3), the angular speed $\omega$ of wheel rotation can be obtained as follows:

$$
\omega=\left(V_{1}-V_{2}\right) / L
$$


Formula (4) shows that the center point velocity $V_{c}$ of the car body is:

$$
V_{c}=\omega R=\left(V_{1}-V_{2}\right) R / L
$$

At the same time, the center point velocity $V_{c}$ of the car body is:

$$
V_{c}=\left(V_{1}+V_{2}\right) / 2
$$

The instantaneous radius $R$ of curvature at the center of formulas (5) and (6) is:

$$
R=\left(V_{1}+V_{2}\right) L / 2\left(V_{1}-V_{2}\right)
$$

Formula (7) shows that when the speed of left and right wheels is equal, the direction is the same, and the radius of rotation tends to be infinite, which can be regarded as linear motion; when the speed of left and right wheels is equal and the direction is opposite, the radius is 0 , and the vehicle rotates in situ; when the speed of left and right wheels are not equal, the intelligent vehicle rotates around a point on the center line of the two wheels.

Assuming that the moving time of intelligent vehicle is very short, the linear speed and rotation angular speed of intelligent vehicle can be considered as a constant.

$$
\begin{aligned}
& \dot{x}=V_{c} \cos \theta \\
& \dot{y}=V_{c} \sin \theta \\
& \dot{\theta}=\omega
\end{aligned}
$$

The constraint equation of the system is:

$$
\dot{x} \sin \theta-\dot{y} \cos \theta=0
$$

From Formula 8 to 11, it can be seen that the direction of the instantaneous speed of the intelligent vehicle is always the same as that of its body (Kim et al. 2018).

Because the difference of two wheels can change the direction of intelligent vehicle, it can be considered that the trajectory of intelligent vehicle is composed of a series of small arcs revolving around the instantaneous center of the circle.

Rational planning of the speed of the left and right wheels of the robot can drive the robot to walk curves of different curvatures.

\subsection{Collision avoidance}

Based on the above principle, a collision avoidance model is designed as shown in Figure 3.

The model combines single-point ultrasonic obstacle avoidance with infrared two-way crossobstacle avoidance to achieve all-round and effective obstacle avoidance.

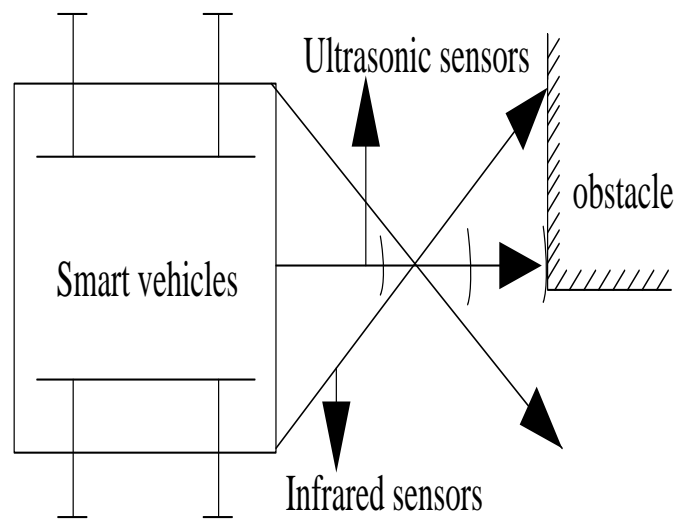

Figure 3 Implementation of obstacle avoidance

For this reason, two infrared obstacle avoidance sensors are installed at the front end of the intelligent vehicle.

They cross-detect obstacles in front of the intelligent vehicle, effectively expanding the detection range, so as to achieve double-sided collision avoidance. In order to prevent smart vehicles from hitting the front, an ultrasonic sensor is set between the two infrared sensors.

When the intelligent vehicle is moving, when the obstacle is detected by the ultrasonic sensor, the intelligent vehicle will turn to the left by default.

When only one side of the infrared sensor detects the obstacle, the intelligent vehicle will turn to the other side without the obstacle.

After turning, when all the sensors no longer detect the obstacle, the intelligent vehicle will go straight again.

There is a looming effect in the driver's perception of the target in front of the intelligent vehicle, that is, the size of the main target projected into the driver's vision depends on the relative distance at this time.

The reciprocal collision time $\left(\mathrm{TTC}^{-1}\right)$ can effectively characterize the above-mentioned "looming" effect, thus reflecting the driver's collision avoidance characteristics.

Its definition is shown in Formula (12), where $V_{c}$ and $V_{p}$ are the velocities of the main car and the front car respectively.

$$
T T C^{-1}=\frac{V_{c}-V_{p}}{R}
$$

Table 2 shows the danger level of intelligent vehicle collision avoidance system. 
Table 2 Hazard rating for intelligent vehicle collision avoidance systems

\begin{tabular}{|c|c|c|c|c|c|c|c|}
\hline \multicolumn{2}{|c|}{ Hazard level } & \multirow{2}{*}{ vision } & \multirow{2}{*}{ hearing } & \multirow{2}{*}{$\begin{array}{c}\text { Active } \\
\text { braking }\end{array}$} & \multicolumn{3}{c|}{ Expected TTC-1 indicator } \\
\cline { 6 - 8 } I level & safety & green & no & no & $<0.35$ & $<0.5$ & $<0.6$ \\
\hline II level & $\begin{array}{c}\text { More } \\
\text { dangerous. }\end{array}$ & yellow & no & no & $0.35-0.5$ & $0.5-0.7$ & $0.6-0.9$ \\
\hline III level & $\begin{array}{c}\text { Very } \\
\text { dangerous. }\end{array}$ & red & $\begin{array}{c}\text { Alarm } \\
\text { frequency is } \\
\text { increasing. }\end{array}$ & no & $0.5-0.7$ & $0.7-0.9$ & $0.9-1.3$ \\
\hline IV level & $\begin{array}{c}\text { Extreme } \\
\text { danger }\end{array}$ & red & no & have & $>0.7$ & $>0.9$ & $>1.3$ \\
\hline
\end{tabular}

When two sensors detect obstacles, the intelligent vehicle steers in the direction of undetected obstacles. When obstacles are detected by three sensors, that is, intelligent vehicles encounter large obstacles in the corner or in front of them. At this time, intelligent vehicles switch to sharp turn mode. Intelligent vehicles default to turn left in situ. If obstacles are no longer detected by sensors in sharp turn, it means that obstacles have been transferred to the right side of intelligent vehicles, intelligent vehicles switch to forward mode and resume straight travel.

\subsection{Design and implementation of information fusion algorithms}

The algorithm analysis process of realizing information fusion in collision avoidance implementation is as follows:

\subsubsection{Observation matching based on Mahalanobis distance}

Mahalanobis distance is a method proposed by Indian statisticians to calculate the covariance distance between two points. The Markov distance between the predicted and observed values is defined as:

$$
d_{M}=\sqrt{\left(z_{i}-z_{k \mid k-1}\right)^{T} S_{k}^{-1}\left(z_{i}-z_{k \mid k-1}\right)}
$$

In the formula, $z_{i}$ is the $i$ target observation value of the current cycle, $z_{k \mid k-1}$ is the target prediction value of the current cycle based on the previous $k-1$ time, $S_{k}$ is the covariance matrix between two samples, and the n-column element of the m-th row is defined as the set of observation values satisfying the covariance conditions of the $m$ and $n$ elements of the two samples.

$$
V_{k}=\left\{z: \sqrt{\left(z_{i}-z_{k \mid k-1}\right)^{T} S_{k}^{-1}\left(z_{i}-z_{k \mid k-1}\right)} \leq C\right\}
$$

In formula: $z$ is an element in the set of observation values. If an observation value is in the region defined by formula (14), it is considered valid.
When $C$ is a limited threshold, the valid observation value will be included in the region when $C$ is 3 . As mentioned in the previous section, the first problem to be solved in the information fusion method in this paper is the matching of observation values. Its mathematical description is as follows:

$$
x_{k}=f_{k}\left(x_{k-1}, u_{k-1}, v_{k-1}\right)
$$

In the Formula: $\left\{x_{k}, k=0,1,2, \cdots\right\}$ is a discretetime sequence of state vectors. $u_{k-1}$ is a known control input (there is no control input in tracking vehicle targets). $\left\{v_{k}, k=0,1,2, \cdots\right\}$ is a system noise which is independent of other variables and satisfies normal distribution.

\subsubsection{Data fusion based on JPDA}

As mentioned in the previous section, after matching the observations, the next step is to fuse them effectively. In this paper, the joint probability data association (JPDA) method is used to fuse the data of ultrasonic sensor and infrared sensor.

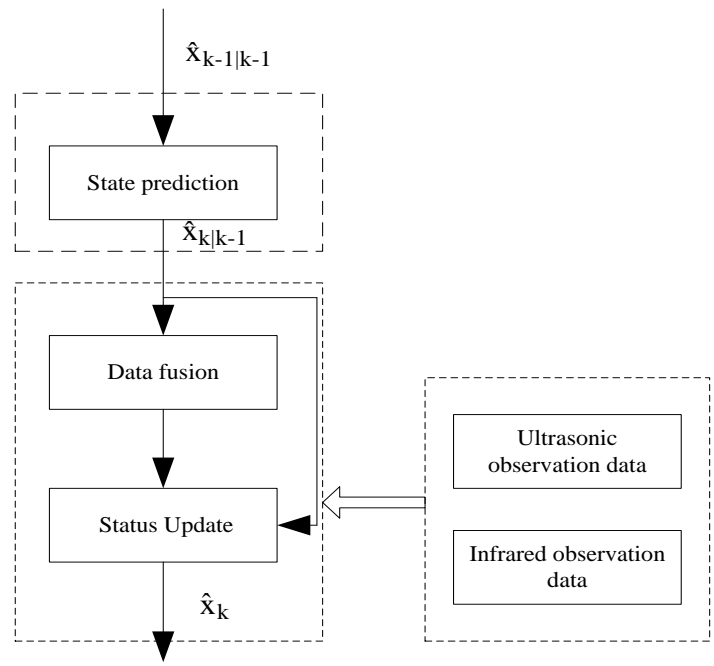

Figure 4: JPDA algorithm flow

Figure 4 illustrates the principle of JPDA state estimation, including target state update and probability of existence update. 
The process of state updating is basically consistent with standard Kalman filter, and the probability of existence is tracked at the same time. In the vehicle tracking problem, the state equation of the system is:

$$
x_{i, k}=F_{k} x_{i, k-1}+v_{k}, i=1,2, \cdots, N
$$

In the formula, $x_{i, k}$ is the state vector of $n_{x}$ dimension, subscript $i$ is the target of $i$, white noise with average $v_{k}$ of 0 is only related to the characteristics of equation of state, and the relationship between the observed value and the real state is satisfied:

$$
z_{i j, k}=H_{j} x_{i, k}+w_{j, k}, j=1,2, \cdots, M
$$

In the formula, $z_{i j, k}$ is the observation value of the $j$ sensor to the $i$ target; $H_{j}$ is the linear approximation of the correlation function $h$ in the mathematical model of the real value and observation value, which is related to different sensor observation models; $w_{j, k}$ is the white noise with the mean of 0 , which is noteworthy that it is related to different sensor models. Its covariance matrix satisfies:

$$
E\left(w_{j, k} w_{j, k}^{T}\right)=B_{j}
$$

After updating the state of step $k-1$, the state value and observation value of step $k-1$ should be predicted.

$$
\left\{\begin{array}{l}
\hat{x}_{i, k \mid k-1}=F_{k} \hat{x}_{i, k-1 \mid k-1} \\
\hat{z}_{i j, k \mid k-1}=H_{j, k} \hat{x}_{i, k \mid k-1}
\end{array}\right.
$$

Then the status update process of this cycle is:

$\hat{x}_{i j, k}=\hat{x}_{i, k \mid k-1}+K_{i j} \gamma_{i j} \quad i=1,2, \cdots, N, j=1,2, \cdots, M$

It is noteworthy that this is the result of state updating for the $i$ target based on the measured values of the $j$ sensor. $K_{i j}$ is the Kalman gain matrix and $\gamma_{i j}$ is the residual vector of the observed and predicted values.

$$
\gamma_{i j, k}=z_{i j}-\hat{z}_{i j, k \mid k-1}
$$

$z_{i j}$ is the observation value of the $i$ target verified in the previous section.

In addition, the covariance of the predicted state is defined as:

$$
P_{i, k \mid k-1}=F_{k} P_{i, k-1 \mid k-1} F_{k}^{T}+Q_{k}
$$

In the formula $S_{i j}$ is the covariance matrix of residuals:

$$
S_{i j}=H_{j} P_{i, k \mid k-1} H_{j}^{T}+R_{j}
$$

Finally, the Kalman gain matrix is calculated.

$$
K_{i j}=P_{i, k \mid k-1} H_{j}^{T} S_{i j}^{-1}
$$

Next, the most important thing is to update the state values, i.e. the weighted average of the predicted states of each observation value, that is:

$$
\hat{x}_{i, k \mid k}=\sum_{j=0}^{M_{i, k}} \beta_{i j} \hat{x}_{i j}
$$

The probability that $\beta_{i j}$ produces the observed value $z_{i j}$ for the target $x_{i}$ in the formula.

The covariance of the state variables is updated to:

$$
P_{i, k \mid k}=\sum_{i=0}^{M_{i, k}} \beta_{i j}\left[P_{i, k \mid k-1}-K_{i j} S_{i j} K_{i j}^{T}\right]+\eta_{i j}
$$

In the formula $\eta_{i j}$ is the hypothesis deviation, which is defined as:

$$
\eta_{i j}=\left(\hat{x}_{i j}-\hat{x}_{i, k \mid k}\right)\left(\hat{x}_{i j}-\hat{x}_{i, k \mid k}\right)^{T}
$$

Because the visual algorithm cannot directly obtain the speed information of the target vehicle, this paper uses the "constant speed model" to track the vehicle. In the discrete state model, the state vector is selected as:

$$
x=\left[\begin{array}{llll}
l^{y} & v^{y} & l^{x} & v^{x}
\end{array}\right]^{T}
$$

The four elements represent the longitudinal position, the longitudinal speed, the lateral position and the lateral speed of the vehicle target respectively. The system matrix $F_{k}$ satisfies:

$$
F_{k}=\left[\begin{array}{cccc}
1 & \Delta t & 0 & 0 \\
0 & 1 & 0 & 0 \\
0 & 0 & 1 & \Delta t \\
0 & 0 & 0 & 1
\end{array}\right]
$$

In addition, the observation vector in the observation model is $z=\left[\begin{array}{ll}l^{y} & l^{x}\end{array}\right]^{T}$. The matrix $H_{j}$ satisfies:

$$
H_{j}=\left[\begin{array}{llll}
1 & 0 & 1 & 0 \\
0 & 0 & 0 & 0
\end{array}\right]
$$

The fusion algorithm in this paper defines that when $C=3$ is used, the valid observations will be included in the description area.

\section{Results}

In order to verify the effectiveness of the collision avoidance technology in this paper, an airport is used as an experimental site to verify the expected braking pressure of a certain type of intelligent vehicle. The actual braking pressure of the experimental vehicle under the control of the collision avoidance technology in this paper is shown in Figure 5. Figure 5(a) and (b) braking pressure response curves for step and sinusoidal desired signals respectively. By comparing the expected braking pressure with the actual braking pressure, it can be seen that the experimental intelligent vehicle controlled by collision avoidance technology can 
quickly and accurately respond to the braking instructions, meet the requirements of the intelligent vehicle collision avoidance system on the brake actuator, and ensure the intelligent vehicle to avoid collision efficiently and accurately.

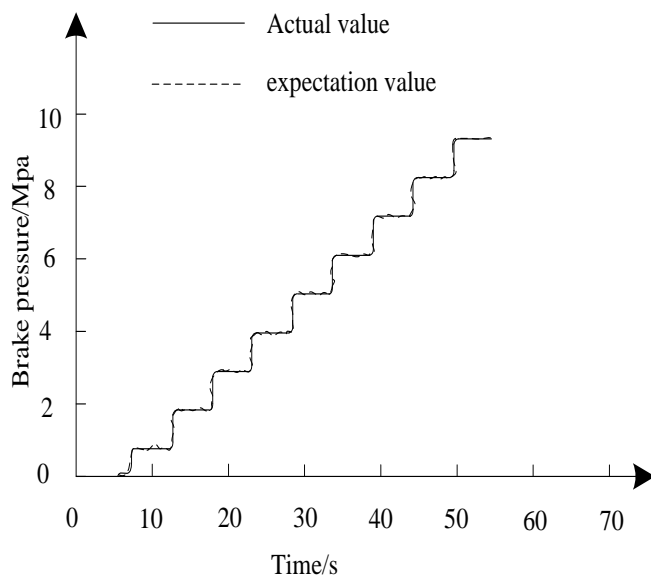

(a) Step expectation signal

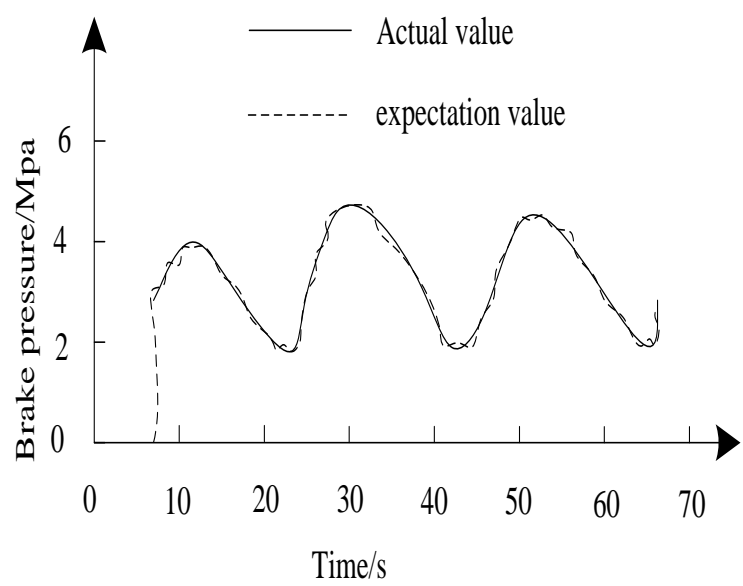

(b) Sine expectation signal

Figure 5: Brake pressure contrast chart

In the experiment, a section of flat ground with many irregular obstacles was selected in the airport.

The obstacles were placed artificially by irregular obstacles, including a certain number of cylindrical obstacles. During the experiment, the intelligent obstacle avoidance vehicle continuously detects whether there are obstacles around the front in the course of moving. When there are obstacles, the position of the obstacles is judged and the corresponding actions are carried out.

Figure 6 is the experimental curve of obstacle avoidance for intelligent vehicles. It can be seen from the graph that when the sensor finds obstacles, the intelligent vehicles turn accordingly.

At this time, because the obstacles are far away, the initial curve oscillation is relatively large. The intelligent vehicle moves forward while steering, and the distance from the obstacle decreases gradually. When it reaches a certain distance, the vehicle avoids the obstacle.
At this time, the sensor cannot detect the obstacle.

When the sensor detects the next obstacle, it judges the position of the corresponding obstacle, and repeats the above actions to cycle.

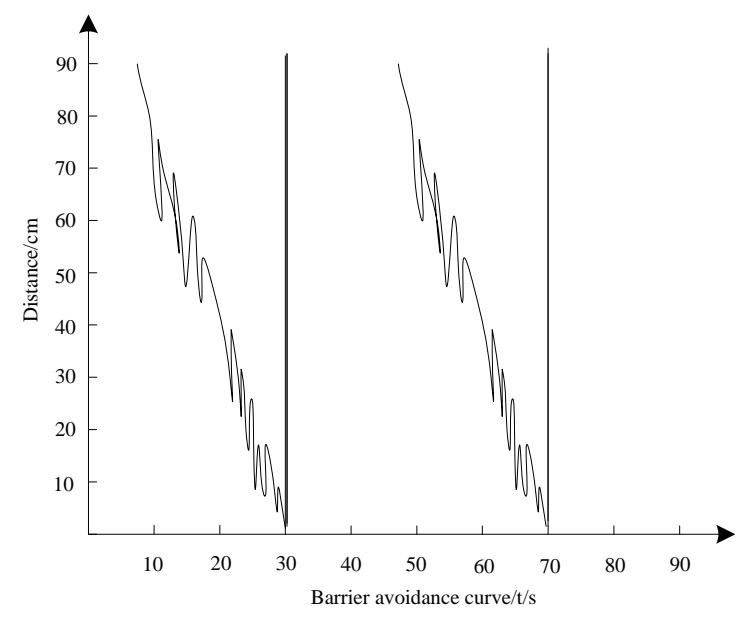

Figure 6: Experimental curve of intelligent vehicle avoidance

In order to validate the success rate of obstacle avoidance of intelligent vehicles effectively, the performance of vehicles is tested by changing the shape of obstacles, and the system is compared with single sensor system. Each vehicle is tested 50 times.

The number of times that one-sided obstacle avoidance and all-round obstacle avoidance succeeded in passing through different obstacle environments was counted.

The obstacle environments consisted of 50 columnar obstacles and surface obstacles. Figure 7 is a successful comparison of obstacle avoidance between single sensor vehicle and multi-sensor vehicle. Obstacle avoidance can be seen from Figure 7 that the common single sensor collision avoidance technology has a lower success rate, while the collision avoidance technology in this paper is not affected by this, and has a higher pass rate.

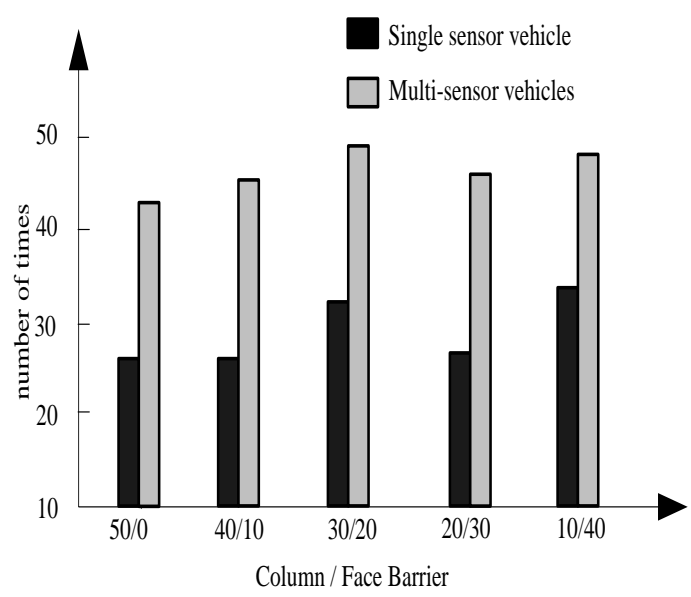

Figure 7: Comparison of barrier avoidance success rate 
The collision avoidance technology is applied to fuse the target identified by the ultrasonic sensor and the infrared sensor in the experiment.

Figures 8 and 9 are the results of target spatial position (horizontal/vertical) state estimation for the same 13 s sample.

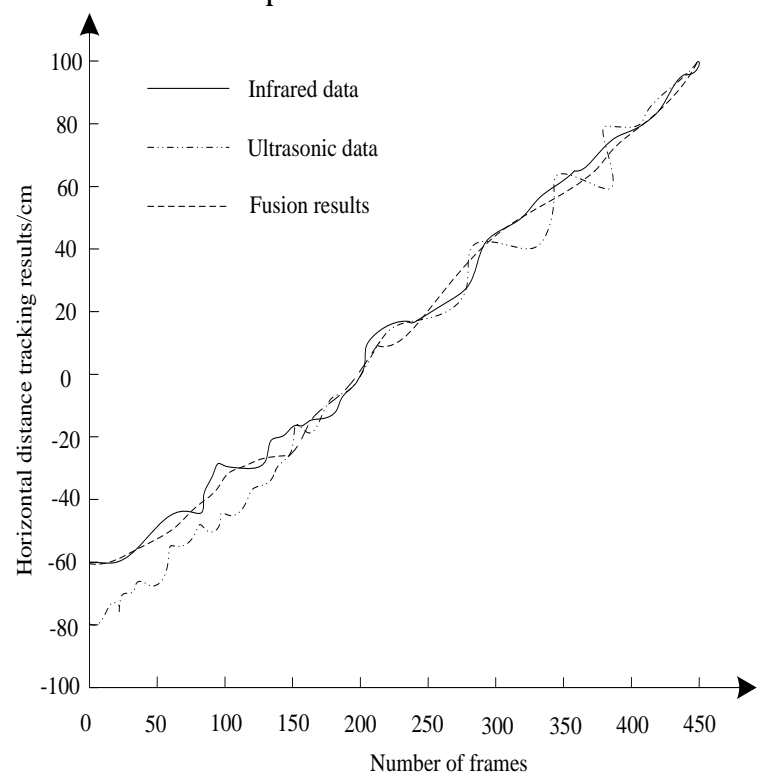

Figure 8: Target transverse distance state estimation results

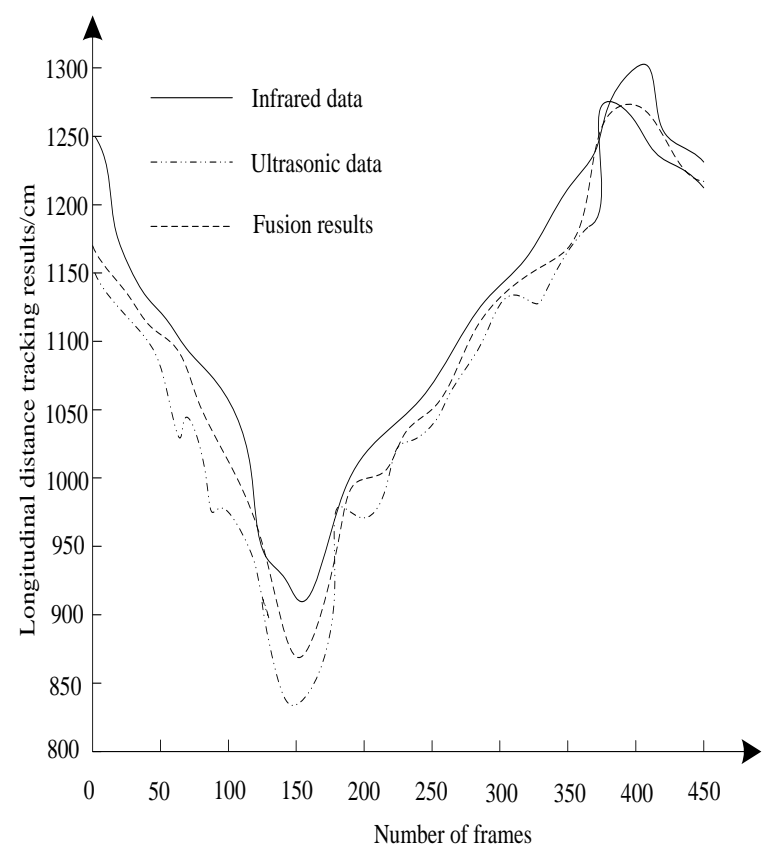

Figure 9: Target longitudinal distance state estimation results

From Figure 8 and Figure 9, we can see that the collision avoidance technology in this paper has better performance of state estimation.

In order to further count the performance of multi-sensor data fusion and recognition based on the collision avoidance technology in this paper, five different performance indicators are counted for over 7000 frames of images: (1) successfully fused targets; (2) only detected by infrared sensors; (3) only detected by ultrasonic sensors; (4) unsuccessful targets; (4) unsuccessful fusion target; (5) Nonvehicle obstacles, usually the guardrail detected by ultrasound.

Table 3 shows the statistical results of the fusion algorithm. As shown in Table 3, the number of unsuccessful fusion is only 4 out of 7000 image samples, accounting for $6 \%$ of the total events. It can be seen that the fusion accuracy of collision avoidance technology in this paper is high, which can make up for the defects of the ultrasonic sensor and the infrared sensor, and identify the target accurately.

Table 3. Statistical results of fusion algorithm

\begin{tabular}{|c|c|c|}
\hline project & number & proportion/\% \\
\hline $\begin{array}{c}\text { Successful } \\
\text { integration }\end{array}$ & 38 & 58 \\
\hline $\begin{array}{c}\text { Ultrasonic } \\
\text { recognition }\end{array}$ & 7 & 11 \\
\hline Infrared recognition & 11 & 17 \\
\hline Failure to integrate & 4 & 6 \\
\hline $\begin{array}{c}\text { Non-vehicle } \\
\text { obstacles }\end{array}$ & 5 & 8 \\
\hline total & 65 & 100 \\
\hline
\end{tabular}

The collision avoidance technology in this paper is experimented on a flat road at the airport. In order to simulate the sudden appearance of obstacles in front of the vehicle when it is running normally, when the test vehicle approaches, cartons are quickly dragged into the main lane as static obstacles in the experimental process. Considering the safety of the experiment, the experiment was carried out only on dry cement road, and conservative driving parameters were set in advance.

Figure 10 shows the test results of automatic collision avoidance technology in this paper. The vehicle was traveling at a constant initial speed of 37 $\mathrm{km} / \mathrm{h}$. At $15.7 \mathrm{~s}$, the radar found obstacles at $34 \mathrm{~m}$ ahead.

As shown in Figure 10(a) and e, when the 16.5 s, the relative vehicle distance starts to be less than the alarm distance, when the risk factor is less than 1 , the sound and light alarm is triggered. Because the driver did not react, when the relative vehicle distance was less than the braking distance and the danger coefficient was less than 0 at 17.4 seconds, the collision avoidance technology in this paper started active braking, and after 17.6 seconds, the vehicle speed decreased significantly, and finally stopped at 2.5 meters away from the obstacle.

Figure 10(b) gives the information of wheel speed and vehicle speed after ABS intervention. In the braking process, the collision avoidance technology actively applied about 6.5 MPa braking 
pressure, resulting in a deceleration of $-6 \mathrm{~m} / \mathrm{s}^{2}$, as shown in Figure 10(c) and(d).

Figure 10 (f) reflects the reciprocal TTC- 1 value of the actual collision time corresponding to the start alarm and active braking time, which are 0.45 and

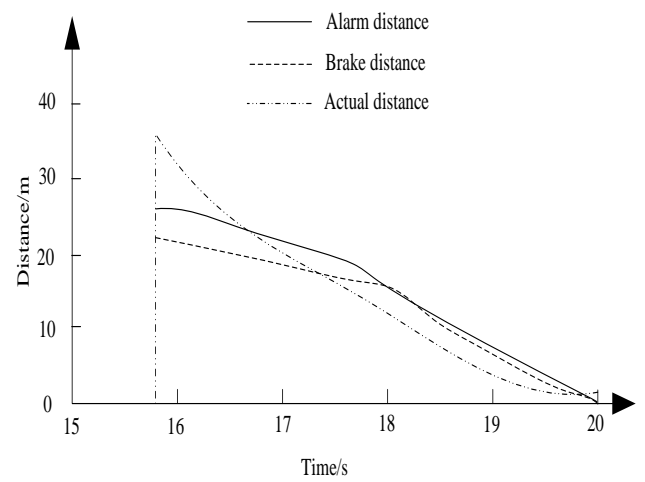

(a) Distance

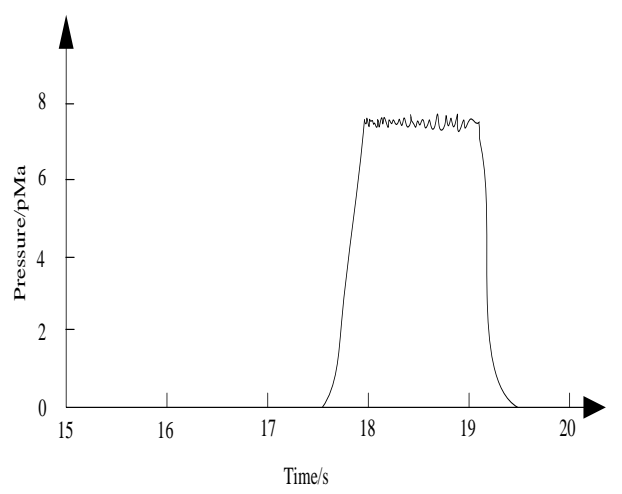

(c) Brake pressure

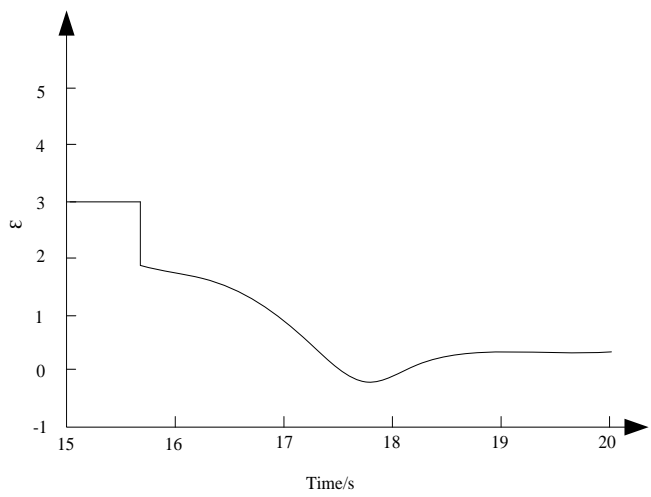

(e) Hazard factor
0.77 respectively. They are within the reasonable range of TTC-1 in Table 2 . It is verified that the collision avoidance technology in this paper meets the system requirements and can effectively avoid obstacles.

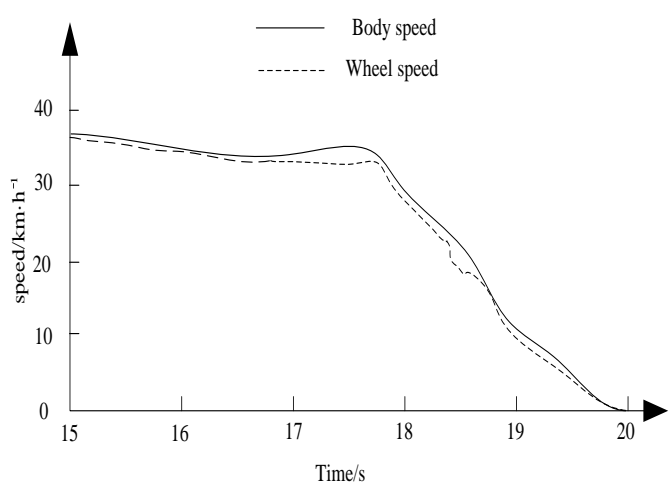

(b) Speed

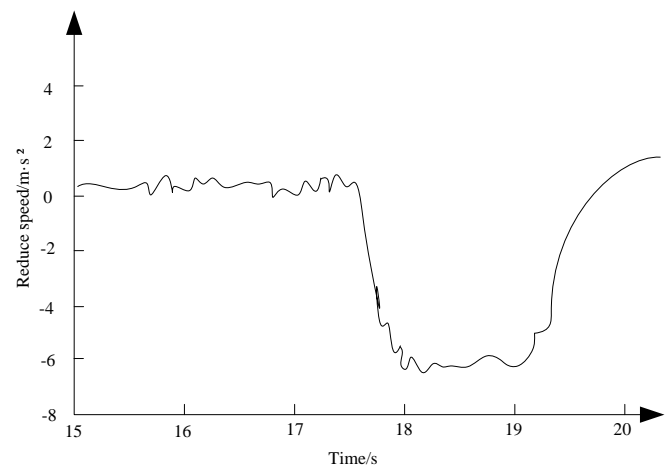

(d) Reduce speed

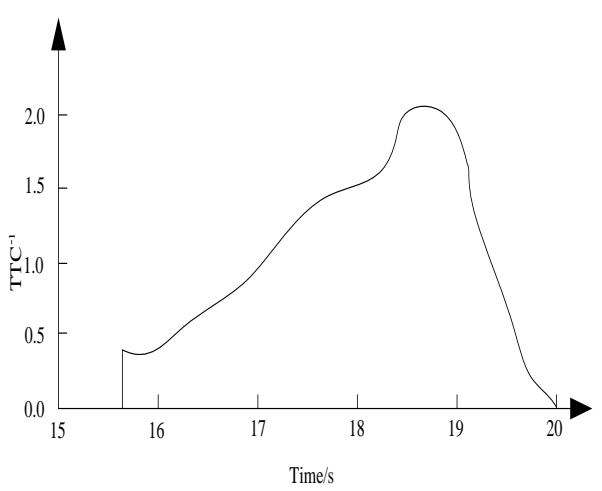

(f) Counting collision time

Figure 10 Automatic collision avoidance test results

\section{Discussions}

The current increase in the number of cars has caused tremendous pressure on traffic, and also posed great challenges to road safety. This paper studies the collision avoidance technology of intelligent vehicle system based on multi-sensor fusion, which has a very positive significance for reducing traffic accidents caused by road environment and subjective reasons, as well as the development of intelligent vehicles. The collision avoidance techniques in this paper are discussed as follows:

(1) Vehicles work in a variety of non-Gaussian interference environments. It is difficult to detect and track vehicle targets accurately using traditional filtering techniques and linear or approximate linear processing methods such as Kalman. In this paper, the joint probabilistic data association JPDA method is used to fuse ultrasonic sensor data with infrared 
sensor data. For non-linear systems and non-linear systems, it is difficult to detect and track vehicle targets accurately. The interference environment of Gauss background has high adaptability. It can solve the problem of low accuracy and even divergence, and overcome the shortcoming of poor reliability of vehicle target tracking. By comparing the braking pressure response curves of the step and sinusoidal expected signals of the intelligent vehicle system under the control of Figure 5 with the expected braking pressure and the actual braking pressure, it is concluded that the technology in this paper can respond to the braking instructions efficiently and accurately, and enhance the collision avoidance performance of the intelligent vehicle.

(2) The technology of this paper focuses on data fusion between infrared sensor and ultrasonic sensor. Mahalanobis distance is introduced to match the observed values of vehicle target sequence.

Through data fusion algorithm based on JPDA, multi-sensor is fused. The tracking accuracy and fusion calculation speed are taken into account, so that infrared sensor and ultrasonic sensor can be perfectly integrated in the process of intelligent vehicle collision avoidance control. By discussing Figure 7 , it can be concluded that the collision avoidance technology in this paper is not affected by the change of obstacle shape, and has a high pass rate. It can be obtained by Table 3. In 7000 image samples, only 4 of the total number of unsuccessful fusion are obtained, accounting for $6 \%$ of the total events. The fusion accuracy of collision avoidance technology in this paper is high, which can solve the defects of the ultrasonic sensor and the infrared sensor, and identify the target accurately. The main reason is that the technology in this paper sets up two infrared obstacle avoidance sensors at the front end of the intelligent vehicle. They cross-detect obstacles in front of the intelligent vehicle, effectively expanding the detection range and realizing double-sided obstacle avoidance. In order to prevent smart vehicles from hitting the front, an ultrasonic sensor is set between the two infrared sensors. When the intelligent vehicle is moving, when the obstacle is detected by the ultrasonic sensor, the intelligent vehicle will turn to the left by default.

When only one side of the infrared sensor detects the obstacle, the intelligent vehicle will turn to the other side without the obstacle. After turning, when all the sensors no longer detect the obstacle, the intelligent vehicle will go straight again.

\section{Conclusions}

In this paper, the collision avoidance technology of intelligent vehicle system based on multi-sensor fusion is studied in depth. The comprehensive collision avoidance technology which combines ultrasonic single-point obstacle avoidance with infrared double-way cross-obstacle avoidance is adopted. Arduino is used as the main control unit and Linux is used as the development platform. Through data acquisition and information fusion control of multi-sensor, the combination of ultrasonic single-point obstacle avoidance and infrared double-way cross-obstacle avoidance is adopted. The mode realizes omni-directional automatic collision avoidance, and solves the problems of obstacle detection difficulty and low success rate of obstacle avoidance in single sensor system.

The experimental results show that the active braking operation of the collision avoidance system accords with the expected TTC- 1 index, and there is no obvious fluctuation in the speed of the vehicle during the control switching, which indicates that the system characteristics match the intelligent vehicle better. The collision avoidance technology in this paper improves the efficiency and success of intelligent vehicle collision avoidance, and can effectively achieve all-round collision avoidance. The actual vehicle test verifies the effectiveness and practicability of the wall crash technology in this paper.

\section{Acknowledgement}

Natural Science Foundation for College and Universities in Jiangsu Province (No. 16KJB520051).

\section{References}

[1] Bila, C., Sivrikaya, F. \& Khan, M. A. 2017. Vehicles of the Future: A Survey of Research on Safety Issues. IEEE Transactions on Intelligent Transportation Systems 18(5): 1046-1065.

[2] Fei, X.J., Li, X. F. 2016. Wireless Sensor Network Data Fusion Algorithm Based on Compressed Sensing Theory. Journal of Jilin University (Science Edition) 54(3): 575-579.

[3] Funke, J., Brown, M. \& Erlien, S. M. 2017. Collision Avoidance and Stabilization for Autonomous Vehicles in Emergency Scenarios. IEEE Transactions on Control Systems Technology 25(4): 1204-1216.

[4] Häusler, A. J., Saccon, A. \& Aguiar, A. P. 2016. Energy-Optimal Motion Planning for Multiple Robotic Vehicles with Collision Avoidance. IEEE Transactions on Control Systems Technology 24(3): 867-883.

[5] Jia, Y., Cebon, D. 2016. Field Testing of a Cyclist Collision Avoidance System for Heavy Goods Vehicles. IEEE Transactions on Vehicular Technology 65(6): 4359-4367.

[6] Jie, J., Khajepour, A. \& Melek, W. W. 2017. Path Planning and Tracking for Vehicle Collision Avoidance Based on Model Predictive Control 
With Multiconstraints. IEEE Transactions on Vehicular Technology 66(2): 952-964.

[7] Johansen. T. A., Perez, T. \& Cristofaro, A. 2016. Ship Collision Avoidance and COLREGS Compliance Using Simulation-Based Control Behavior Selection with Predictive Hazard Assessment. IEEE Transactions on Intelligent Transportation Systems 17(12): 3407-3422.

[8] Kim, Y. H., Park, J. B. \& Yoon, T. S. 2018. Modified turn algorithm based ground vehicle collision avoidance. Electronics Letters 54(9): 558-560

[9] Li, J. 2017. Design of communication power supply monitoring and management system based on multimedia technology. Chinese Iournal of Power Sources 41(4): 635-637.

[10] Li, P., Xing, W. 2016. Application of Information Fusion Technology in Sensor Network. Automation \& Instrumentation (7): 202-203.

[11] Lin, Y., Saripalli, S. 2017. Sampling-Based Path Planning for UAV Collision Avoidance. IEEE Transactions on Intelligent Transportation Systems 18(11): 3179-3192.
[12] Lu, Z. J., Liu, X. \& Qin, Y. G. 2017. An Adaptive Multi-sensor Management Method for Cooperative Perception. Lournal of China Academy of Electronics and Information Technology 12(4): 353-358.

[13] Mylvaganam, T., Sassano, M. \& Astolfi, A. 2017. A Differential Game Approach to Multi-agent Collision Avoidance. IEEE Transactions on Automatic Control 62(8): 4229-4235.

[14] Rong, D. S., Hu, J. S. \& Zhao, J. J. 2018. Prediction Model of Methane Yield from Low-rank Coal Based on Data Fusion and IGA-RGRNN Algorithm. Journal of power supply 16(1): 178184.

[15] Sabattini, L., Secchi, C. \& Fantuzzi, C. 2017. Coordinated Dynamic Behaviors for Multirobot Systems With Collision Avoidance. IEEE Transactions on Cybernetics 47(12): 40624073.

[16] Zheng Y. J., Tian K. S. \& Zhang, J. L. 2017. SelfAdaptive Management Method of Multi-Sensor Resource Based on Efficacy Function. Computer Simulation 34(5): 322-326. 